TAMKANG JOURNAL OF MATHEMATICS

Volume 21, Number 1, Spring 1990.

\title{
LOCAL DISCRETE EXTENSIONS OF SUPRATOPOLOGIES
}

\author{
M.E. ABD El-MONSEF AND E.F. LASHIEN
}

\begin{abstract}
A.bstract. In this paper, we introduce the concept of local discrete extensions of supratopologies on a set. The basic problem is to investigate the supratopological properties that are preserved under local discrete extensions.
\end{abstract}

\section{Introduction}

Let $(X, \tau)$ be a topological space on which no separation axioms are stated and whenever such axioms are needed they will be explicitly assumed. A class $\tau^{*} \subset P(X)$ is called a supratopology [2] on $X$ if $X \in \tau^{*}$ and $\tau^{*}$ is closed under arbitrary union. $\left(X, \tau^{*}\right)$ is called supratopological space (briefly a supraspace). A supratopology $\tau^{*}$ is called associated with $\tau$ if $\tau \subset \tau^{*}$ and each member of $\tau^{*}$ is called a supraopen set and the complement of a suparopen set is called supraclosed [2]. Various notions like interior, closure, exterior and the derived set operators can be defined in supratopological spaces in analogy with topological spaces [2]. The supraderived set (resp. supraclosure, suprainterior) of a subset $A$ of a space $X$ will be denoted by $d_{\tau^{*}} A$ (resp. $c l_{\tau^{*}} A, i n t_{\tau^{*}} A$ ). Also, Mashhour, et. al [2] have introduced the concept of $S-T_{i}(i=0,1,2)$ and $S-T_{2}^{\prime}$ separation axioms in supraspaces, by replacing open sets by supraopen sets in $T_{i}$ and $T_{2}^{\prime}$ separation axioms. By the same manner they introduced the concept of $S^{*}$-regularity and $S^{*}$-normality [3]. In [2] the concept of $S^{*}$-continuity was defined as follows : A function $f:\left(X, \tau_{1}^{*}\right) \rightarrow\left(Y, \tau_{2}^{*}\right)$ is $S^{*}-$ continuous if the inverse image of each $\tau_{2}^{*}$-supraopen set is $\tau_{1}^{*}$ supraopen.

In 1971, Young, S. P. [4] introduced the concept of local discrete extensions of topologies. Let $(X, \tau)$ be a topological space and $A$ be a subset of $X$. Then the topology $\tau[A]=\{U-B: U \in \tau, B \subset A\}$ is called local discrete extension of $\tau$ by $A$. He attampted to investigate, if $(X, \tau)$ has some topological property $Q$, under what conditions will $(X, \tau[A])$ also have property $Q$.

The purpose of the present paper is to introduce the concept of local discrete extensions of supratopologies on a set and to study the preservation of some supratopological properties under local discrete extensions, in a way analogous to results obtained by Young [4]. Also, we introduce and study the concept of the base for supratopologies, the local base at a point in a supraspaces, the first countable and the second contable

Received September 21, 1987; revised Appil 11, 1989. 
supraspaces and study the preservation of such supraspaces under local discrete extensions.

\section{Local discrete extensions of supratopologies}

Definition 2.1. Let $\left(X, \tau^{*}\right)$ be supraspace and $A \subset X$, then $\tau^{*}[A]$ is called local discrete extension of $\tau^{*}$ by $A$ iff $\tau^{*}[A]=\left\{U-B: U \in \tau^{*}, B \subset A\right\}$.

It is clear that $\tau^{*}[A]$ is a supratopology on $X$ and $\tau^{*} \subset \tau^{*}[A]$.

Remark 2.1. (i) If $\tau$ is topology on $X$ and $\tau^{*}$ is an associated supratopology with $\tau$, then $\tau[A] \subset \tau^{*}[A]$, where $\tau[A]$ is the local discrete extension of $\tau$ by $A$ in the sense of Young [4]. The inclusion relation cannot be replaced by equality sign, in general, as shown by Example 2.1.

(ii) The concept of local discrete extensions and simple extensions of supratopologies [1] are independent concepts (Example 2.2).

Example 2.1. Let $X=\{a, b, c, d\}$ with topology $\tau=\{X, \phi,\{a\}\}$ and supratopology $\tau^{*}=\{X, \phi,\{a\},\{a, c\},\{b, c\},\{a, b, c\}\}$, For $A=\{b, d\}, \tau[A]=\{X, \phi,\{a\},\{a, c\}$, $\{a, b, c\},\{a, c, d\}\}$ and $\tau^{*}(A)=\{X, \phi,\{a\},\{a, c\},\{a, b, c\},\{a, c, d\},\{c\},\{b, c\}\}$. Therefore, $\tau[A] \neq \tau^{*}[A]$.

Example 2.2. Let $X=\{a, b, c\}$ with topology $\tau=\{X, \phi,\{a\},\{a, c\}\}$ and supratopology $\tau^{*}=\{X, \phi,\{a\},\{a, c\},\{b, c\}\}$. For $A=\{a, b\}, \tau^{*}[A]=\{X, \phi,\{a\},\{a, c\}$, $\{b, c\},\{c\}\}$ and $\tau^{*}(A)=\{X, \phi,\{a\},\{a, c\},\{b, c\},\{a, b\},\{b\}\}$.

Proposition 2.1. If $\left(X, \tau^{*}\right)$ is a supraspace and $A \subset X$, then $\left(A, \tau^{*}[A] \cap A\right)$ is discrete.

Proof. To prove that $\left(A, \tau^{*}[A] \cap A\right)$ is discrete for any $A \subset X$, we need to show that every singletion $\{p\} \subset A$ is both open and closed in $\left(A, \tau^{*}[A] \cap A\right)$. Let $\{p\} \subset A$. Then $X-\{p\} \in \tau^{*}[A]$ and $(X-\{p\}) \cap A=A-\{p\} \in \tau^{*}[A] \cap A$ and hence $\{p\}$ is supraclosed in $A$. On the other hand, $A-\{p\} \subset A$ implies $X-(A-\{p\}) \in \tau^{*}[A]$ and $X-(A-\{p\}) \cap A=A-(A-\{p\})=\{p\} \in \tau^{*}[A] \cap A$.

Proposition 2.2. For a supraspace $\left(X, \tau^{*}\right), \tau^{*}[A] \supset \tau^{*}[B]$ for any $B \subset A$.

Proof. $\tau^{*}[B]=\left\{U-C: U \in \tau^{*}, C \subset B \subset A\right\} \subset \tau^{*}[A]$. The inclusion relation in Proposition 2.2 cannot be replaced by equality sign as shown by the following example.

Example 2.3. Let $X=\{a, b, c, d\}$ with topology $\tau=\{X, \phi,\{a\},\{a, d\}\}$ and supratopology $\tau^{*}=\{X, \phi,\{a\},\{a, d\},\{a, c\},\{a, c, d\}\}$. For $A=\{a, b, d\}$ and $B=\{b\} \subset$ $A, \tau^{*}[A]=\{X, \phi,\{a\},\{a, d\},\{a, c\},\{a, c, d\},\{c\},\{d\},\{c, d\}\}$ and $\tau^{*}[B]=\{X, \phi,\{a\}$, $\{a, d\},\{a, c\},\{a, c, d\}\}$.

Theorem 2.1. If $\left(X, \tau^{*}\right)$ is a supraspace, $\tau^{*}[A]$ is a local discrete extension of $\tau^{*}$ and $B$ is any subset of $X$, then 
(i) $c l_{\tau *[A]} B=(A \cap B) \cup c l_{\tau} \cdot((X-A) \cap B)$.

(ii) int $_{\tau^{*}[A]} B=((X-A) \cap B) \cap$ int $_{\tau^{*}}(A \cup B)$.

(iii) $d_{\tau^{*}[A]} B \subset d_{\tau^{*}} B\left(d_{\tau^{*}[A]}, d_{\tau^{*}}\right.$ denote the derived operator relative to $\tau^{*}[A]$ and $\tau^{*}$, respectively).

Proof. Proofs of (i) and (ii) follows in a similar manner to the topological case considered by Young [4]. (iii) follows from $\tau^{*} \subset \tau^{*}[A]$.

The inclusion relation in ((iii) Theorem 2.1) cannot be replaced by equality sign as illustrated by the following example.

Example 2.4. In Example 2.3, consider $B=\{a, c\}$, then $d_{\tau^{*}[A]} B=\{b\}$ and $d_{r^{*}} B=\{b, c, d\}$. Hence $d_{\tau^{*}[A]} B \not \supset d_{\tau^{*}} B$.

\section{Preservation of some supratopological properties under local discrete extensions of supratopologies}

In what follows we discuss the preservation of some supratopological properties, $S-T_{i}(i=0,1,2), S-T_{2}^{\prime}$ axioms, $S^{*}$-regularity and $S^{*}$-normality, under local discrete extensions of supratopologies.

Theorem 3.1. If $\left(X, \tau^{*}\right)$ is $S-T_{2}$ (resp. $\left.S-T_{1}, S-T_{0}\right)$ supraspace, then $\left(X, \tau^{*}[A]\right)$ is $S-T_{2}$ (resp. $\left.S-T_{1}, S-T_{0}\right)$.

Proof. Obvious, since $\tau^{*} \subset \tau^{*}[A]$.

The converse of Theorem 3.1 is false, in general, as shown by the following example.

Example 3.1. Let $X=\{a, b, c\}$ with topology $\tau=\{X, \phi,\{a\},\{b, c\}\}$ and supratopology $\tau^{*}=\{X, \phi,\{a\},\{b, c\},\{a, c\}\}$. For $A=\{c\}, \tau^{*}[A]=\{X, \phi,\{a\},\{b, c\}$, $\{a, c\},\{a, b\},\{b\}\} .\left(X, \tau^{*}[A]\right)$ is an $S-T_{2}$ supraspace while $\left(X, \tau^{*}\right)$ is not.

Theorem 3.2. If $\left(X, \tau^{*}\right)$ is $S-T_{2}^{\prime}$, then $\left(X, \tau^{*}[A]\right)$ is $S-T_{2}^{\prime}$.

Proof. Let $\left(X, \tau^{*}\right)$ be an $S-T_{2}^{\prime}$ supraspace and $x, y$ be two distinct points of $X$. Then there exist two supraopen sets $U, V \in \tau^{*} \subset \tau^{*}[A]$ containing $x$ and $y$, respectively, such that $c l_{\tau^{*}} U \cap c l_{\tau^{*}} V=\emptyset$. Hence, $c l_{\tau^{*}[A]} U \cap c l_{\tau^{*}[A]} V \subset c l_{\tau^{*}} U \cap c l V_{\tau^{*}}=\emptyset$ and $\left(X, \tau^{*}[A]\right)$ is an $S-T_{2}^{\prime}$ supraspace.

Theorem 3.3. If $\left(X, \tau^{*}\right)$ is $S^{*}$-regular $\left(S^{*}\right.$-normal) and $A$ is a supraopen subset of $X$, then $\left(X, \tau^{*}[A]\right)$ is $S^{*}$-regular $\left(S^{*}\right.$-normal).

Proof. Let $A$ be a supraopen subset of $\left(X, \tau^{*}\right)$ then every subset $A$ is $\tau^{*}[A]$ supraopen set. Let $F$ be a supraclosed subset of $\left(X, \tau^{*}[A]\right)$ and $x \notin F$. Then there exists a $\tau^{*}[A]$ supraopen set $U-B, U \in \tau^{*}, B \subset A$, such that $F=X-(U-B)=$ $(X-U) \cup(X \cap B)=(X-U) \cup B, x \notin F$. Hence $x \notin X-U$ and $x \notin B$. There 
are two cases (i) $x \notin A$. Since $\left(X, \tau^{*}\right)$ is $S^{*}$-regular, for each $x \notin X-U$, there exist disjoint supraopen sets $U$ and $V$ such that $x \in U$ and $X-U \subset V$. Hence there are disjoint $\tau^{*}[A]$ supraopen sets $U-A$ and $V \cap U$ such that $x \in U-A$ and $F \subset V \cap B$. (ii) $x \in A$. Since $\left(X, \tau^{*}\right)$ is $S^{*}$-regular, there exist disjoint $\tau^{*}$-supraopen sets, consequently $\tau^{*}[A]$ supraopen, $A$ and $V$ such that $x \in A$ and $X-A \subset V$. Therefore, $\left(X, \tau^{*}[A]\right)$ is $S^{*}$-regular.

In case that $A$ is not supraopen subset of $\left(X, \tau^{*}\right)$, the above theorem does not hold in general.

Example 3.2. Let $X=\{a, b, c\}$ with indiscrete supratopology $\tau^{*}=\{X, \phi\}$. Then for $A=\{a, b\}, \tau^{*}[A]=\{X, \phi,\{c\},\{a, c\},\{b, c\}\}$ and hence $\left(X, \tau^{*}\right)$ is $S^{*}$-regular and $S^{*}$-normal while $\left(X, \tau^{*}[A]\right)$ is neither.

\section{Bases for supratopologies}

Definition 4.1. Let $\left(X, \tau^{*}\right)$ be a supraspace. A class $\beta^{*}$ of supraopen sets of $X$, i.e. $\beta^{*} \subset \tau^{*}$ is a base for the supratopology iff every supraopen set $G \in \tau^{*}$ is the union of numbers of $\beta^{*}$, equivalently, for any point $p \in G, G \in \tau^{*}$, there exists a member $B_{p} \in \beta^{*}$ such that $p \in B_{p} \subset G$. Clearly in any supraspace $\left(X, \tau^{*}\right), \tau^{*}$ is a base for itself.

Theorem 4.1. Let $\beta^{*}$ be a class of subsets of a nonempty set $X$. Then $\beta^{*}$ is a base for some supratopology $\tau^{*}$ on $X$ iff $X=\cup\left\{B: B \in \beta^{*}\right\}$.

Proof. Suppose $\beta^{*}$ is a base for a supratopology $\tau^{*}$ on $X$. Since $X$ is supraopen, $X$ is the union of members of $\beta^{*}$, i.e. $X=\cup\left\{B: B \in \beta^{*}\right\}$. Conversely, let $\beta^{*}$ be a class of subsets of $X$ satisfy $X=\cup\left\{B: B \in \beta^{*}\right\}$. Let $\tau^{*}$ be the class of all subsets of $X$ which are unions of members of $\beta^{*}$. We have prove that $\tau^{*}$ is a supratopology an $X$. Since $X=\cup\left\{B: B \in \beta^{*}\right\}, X \in \tau^{*}$. Also, $\phi$ is the union of an empty subclass of $\beta^{*}$, i.e. $\phi=\cup\left\{B: B \in \phi \subset \beta^{*}\right\}$, hence $\phi \in \tau^{*}$. Moreover let $\left\{G_{i}\right\}$ be a class of members of $\tau^{*}$. By definition of $\tau^{*}$, each $\cup_{i} G_{i}$ is the union of members of $\beta^{*}$, hence the union $\cup_{i} G_{i}$ is also a union of members of $\beta^{*}$. So, $\cup_{i} G_{i} \in \tau^{*}$. Therefore, $\tau^{*}$ is a supratopology on $X$.

Theorem 4.2. If $\beta$ is a base for a supratopology $\tau^{*}$ on $X$ and $\beta^{*}$ is a class of supraopen sets containing $\beta$, i.e. $\beta \subset \beta^{*}$. Then $\beta^{*}$ is a base for $\tau^{*}$.

Proof. Let $G$ be a supraopen subset of $X$. Since $\beta$ is a base for $\tau^{*}, G$ is the union of members of $\beta$, i.e. $G=\cup_{i} B_{i}$ where $B_{i} \in \beta$. But $\beta \subset \beta^{*}$, hence each $B_{i} \in \beta$, is also belongs to $\beta^{*}$. So $G$ is the union of members of $\beta^{*}$ and therefore, $\beta^{*}$ is also a base for $r^{*}$.

Definition 4.2. A class $\beta_{p}^{*}$ of supraopen subsets of a supraspace $\left(X, r^{*}\right)$ containing $p \in X$ is called a local base at $p$ iff for each supraopen set $G$ containing $p$, there exists $G_{p} \in \beta_{p}^{*}$ such that $p \in G_{p} \subset G$.

Theorem 4.3. A point $p \in X$ belongs to the supraderived set of $A \subset X\left(p \in d_{\tau^{*}} A\right)$ iff each member of some local base $\beta_{p}^{*}$ at $p$ contains some points of $X$ deifferent from $p$. 
Proof. Let $p \in d_{\tau^{*}} A$, then $(G-\{p\}) \cap A \neq \emptyset$ for all $G \in \tau^{*}, p \in G$. But $\beta_{p}^{*} \subset \tau^{*}$, so inparticular $(B-\{p\}) \cap A \neq \emptyset$ for all $B \in \beta_{p}^{*}$. Conversely, suppose $(B-\{p\}) \cap A \neq \emptyset$ for all $B \in \beta_{p}^{*}$ and let $G$ be any supraopen subset of $X$ containing $p$. Then there exists $B_{0} \in \beta_{p}^{*}$ for which $p \in B_{0} \subset G$. Then $(G-\{p\}) \cap A \supset\left(B_{0}-\{p\}\right) \cap A \neq \emptyset$. So, $(G-\{p\}) \cap A \neq \emptyset$ and hence $p \in d_{\tau^{*}} A$.

Definition 4.3. Let $\left(X, \tau^{*}\right)$ be a supraspace. A sequence $\left\langle a_{n}\right\rangle, n \in N S^{*}-$ converges to a point $b \in X$ iff for each supraopen set $G$ containing $b$, there exists $n_{0} \in N$ such that for all $n \geq n_{0}$ implies $a_{n} \in G$, i.e. if $G \in \tau^{*}$ contains almost all, i.e. all except finite member of the terms of the sequence.

Theorem 4.4. A sequence $\left\langle a_{n}\right\rangle$ of points in a supraspace $\left(X, \tau^{*}\right) S^{*}$-converges to $p \in X$ iff each members of some local base $\beta_{p}^{*}$ at $p$ contains almost all the terms of the sequence.

Proof. $<a_{n}>S^{*}$-converges to $p \in X$ iff every supraopen set $G$ containing $P$ contains almost all the terms of the sequence. But $\beta_{p}^{*} \subset \tau^{*}$, so inparticular each $B \in \beta_{p}^{*}$ contains almost all the terms of the sequence. Conversely, suppose every $B \in \beta_{p}^{*}$ contains almost all the terms of the sequence and let $G$ be any supraopen set containing $p$. Then there exists $B_{0} \in \beta_{P}^{*}$ for which $p \in B_{0} \subset G$. Hence $G$ is also contains almost all the terms of the sequence. Therefore, $\left\langle a_{n}\right\rangle S^{*}$-converges to $p$.

\section{Preservation of first countable and second countable supraspaces under local discrete extensions of supratopologies}

Defimition 5.1. A supraspace $\left(X, \tau^{*}\right)$ is a first countable supraspace iff there exists a countable local base at every $x \in X$.

Definition 5.2. A supraspace $\left(X, \tau^{*}\right)$ is a second countable supraspace iff there exists a countable base $\beta^{*}$ for the supratopology.

Theorem 5.1. If $f:\left(X, \tau^{*}\right) \stackrel{\text { onto }}{\rightarrow}\left(Y, U^{*}\right)$ is an $S^{*}$-continuous mapping and $\left(X, \tau^{*}\right)$ is a second (first) countable supraspace, then $\left(Y, U^{*}\right)$ is a second (first) countable supraspace.

Proof. We prove the theorem only for the second countable supraspace.

Let $\left(X, \tau^{*}\right)$ be a second countable supraspace and let $G$ be a supraopen subset in $\left(Y, U^{*}\right)$. Then $f^{-1}(G) \in \tau^{*}$, because $\mathrm{f}$ is $S^{*}$-continuous, and hence $f^{-1}(G)=\cup\left\{H_{\lambda}: \lambda \in\right.$ $\Lambda \subset N\}$ where $N$ is a countable index set and the family $\left\{H_{m}, m \in M\right\}$ is a countable base for $\tau^{*}$. Since $f$ is onto, $f f^{-1}(G)=G$ and $G=\cup\left\{f\left(H_{\lambda}\right)\right\}$, it follows that the countable fami'y of supraopen sets $\left\{f\left(H_{m}\right), m \in M\right\}$ is a base for $U^{*}$.

Theorem 5.2. If $A$ is a subset of a second countable supraspace $\left(X, \tau^{*}\right)$, then every supraopen cover of $A$ is reducible to a countable cover. 
Proof. Let $\mathcal{U}=\left\{U_{\lambda}, \lambda \in \Lambda\right\}$ be a supraopen cover of $A$, i.e. $A \subset \cup\{U: U \in \mathcal{U}\}$ and let $\beta$ be a countable base for $X$. Hence for $x \in A$, there exists $U_{x} \in \mathcal{U}$ such that $x \in U_{x}$. Since $\beta$ is a base for $\tau^{*}$, for every $x \in A$, there exists $B_{x} \in \beta$ such that $x \in B_{x} \subset U_{x}$. Hence $A \subset \cup\left\{B_{x}: x \in A\right\}$. But $\left\{B_{x}: x \in A\right\} \subset \beta$, so it is countable, hence $\left\{B_{x}: x \in A\right\}=\left\{B_{n}, n \in N\right\}$ where $N$ is a countable index set. For each $n \in N$ choose one set $U_{n} \in \mathcal{U}$ such that $B_{n} \subset U_{n}$. Then $A \subset\left\{B_{n}: n \in N\right\} \subset \cup\left\{U_{n}: n \in N\right\}$. Therefore, $\left\{U_{n}, n \in N\right\}$ is a countable cover of $\mathcal{U}$.

The following theorems discuss the preservation of the first countable and the second countable supraspace under local discrete extensions of supratopologies.

Theorem 5.3. If $A$ is any subset of a supraspace $\left(X, \tau^{*}\right)$, then $\left(X, \tau^{*}\right)$ is a first countable iff $\left(X, \tau^{*}[A]\right)$ is a first countable surpraspace.

Proof. Let $\left\{U_{i}, i=1,2, \ldots\right\}$ be a countable local base at any point $x$ of $\left(X, \tau^{*}\right)$. There are two cases. (i) $x \notin A$. Then $\left\{U_{i}-A, i=1,2, \ldots\right\}$ is a countable local base of a point $x$ of $\left(X, \tau^{*}[A]\right)$. (ii) $x \in A$. Then $\left\{U_{i}-(A-\{x\}): i=1,2, \ldots\right\}$ is a countable local base of a point $x$ of $\left(X, \tau^{*}[A]\right)$. Hence $\left(X, \tau^{*}[A]\right)$ is a first countable supraspace.

Theorem 5.4. If $\left(X, \tau^{*}\right)$ is a second countable supraspace, then $\left(X, \tau^{*}[A]\right)$ is a second countable if $A$ is a countable subset of $\left(X, \tau^{*}\right)$.

Proof. Let $\mathcal{U}$ be a countable base for $\left(X, \tau^{*}\right)$ and $\mathcal{U}_{A}=\left\{B-A_{\alpha}: B \in \mathcal{U}, A_{\alpha}\right.$ is a cofinite subset of $\mathbb{A}\}$. Then $\mathcal{U}_{A}$ is countable and a base for $\tau^{*}[A]$. For, let $x \in B-A_{1}$, and $A_{1} \subset A$. If $A_{1}$ is a cofinite subset in $A$, the proof follows directly. If $A_{1}$ is not cofinite subset in $A$, then $A-A_{1}$ is not finite and we have two cases (i) $x \notin A$. Then $x \notin B-A \subset B-A_{1}$. (ii) $x \in A$. Then $x \in B-(A-\{x\}) \subset B-A_{1}$. Therefore, $\left(X, \tau^{*}[A]\right)$ is a second countable supraspace.

\section{Reference}

[1] Abd El-Monsef, M. E. ; Lashien, E. F., "Simple extensions of supratopologies", Bull. Fac. Sci. Alex. Uni., $27 \mathrm{~A}(1), 1987,35-47$.

[2] Mashhour, A.S., Allam, A. A., Mahmoud, F. S., Kheder, F. H., "On supratopological spaces", Indian, Pure. Apple. Math., 14(4) April 1983, 502-510.

[3] Mashhour, A.S., Allam, A. A., Hasanein, I. A., "Some supratopological results", Johanness Kebeler Univ. Linz. Austria. Math. J. 265, 1983, 1-16.

[4] Young, S. P., "local discrete extensions of topologies, Kyungpook Math. J. II", 1971, 21-24. 